\title{
Characteristics of invasive Acinetobacter species isolates recovered in a pediatric academic center
}

\author{
Avish L. Jain ${ }^{1 \dagger}$, Christian M. Harding ${ }^{1,2 \dagger}{ }^{\dagger}$, Kaivon Assani ${ }^{1}$, Chandra L. Shrestha ${ }^{1}$, Mercedees Haga', Amy Leber ${ }^{3}$, \\ Robert S. Munson Jr. ${ }^{1}$ and Benjamin T. Kopp ${ }^{1,4^{*}}$
}

\begin{abstract}
Background: Acinetobacter species are associated with increasing mortality due to emerging drug-resistance. Pediatric Acinetobacter infections are largely undefined in developed countries and clinical laboratory identification methods do not reliably differentiate between members of the Acinetobacter calcoaceticus-baumannii complex, leading to improper identification. Therefore we aimed to determine risk factors for invasive Acinetobacter infections within an academic, pediatric setting as well as defining microbiologic characteristics of predominant strains.

Methods: Twenty-four invasive Acinetobacter isolates were collected from 2009-2013. Comparative sequence analysis of the $r p o B$ gene was performed coupled with phenotypic characterization of antibiotic resistance, motility, biofilm production and clinical correlation.

Results: Affected patients had a median age of 3.5 years, and $71 \%$ had a central catheter infection source. rpoB gene sequencing revealed a predominance of A. pittii (45.8\%) and A. baumannii (33.3\%) strains. There was increasing incidence of $A$. pittii over the study. Two fatalities occurred in the A. pittii group. Seventeen percent of isolates were multi-drug resistant. A pittii and A. baumannii strains were similar in motility, but A pittii strains had significantly more biofilm production ( $P$ value $=0.018)$.

Conclusions: A. pittii was the most isolated species highlighting the need for proper species identification. The isolated strains had limited acute mortality in children, but the occurrence of more multi-drug resistant strains in the future is a distinct possibility, justifying continued research and accurate species identification.
\end{abstract}

Keywords: Antibiotic resistance, Bacteremia, Pediatric, Acinetobacter

\section{Background}

Acinetobacter species are Gram-negative coccobacilli many of which are found in soil and fresh water throughout our natural habitats [1]. However, certain Acinetobacter species are frequently isolated increasingly from healthcare facilities and are concomitantly the source of many nosocomial infections [1]. Specifically, A. baumannii, A. pittii, and A. nosocomialis of the Acinetobacter calcoaceticus-baumannii (Acb) complex have

\footnotetext{
* Correspondence: Benjamin.Kopp@NationwideChildrens.org

${ }^{\dagger}$ Equal contributors

${ }^{1}$ Center for Microbial Pathogenesis, The Research Institute at Nationwide

Children's Hospital, Columbus, OH, USA

${ }^{4}$ Nationwide Children's Hospital, Section of Pulmonary Medicine, 700

Children's Drive, Columbus, OH 43205, USA

Full list of author information is available at the end of the article
}

become the most medically relevant members of the genus as they are most frequently isolated from health care facilities as well as human tissues. Patients with impaired host defenses in intensive care unit (ICU) settings appear to be an at risk group of acquiring Acinetobacter infections [2]. Despite this knowledge, the infection source in outbreaks often cannot be determined, leaving recommendations to prevent future outbreaks limited [3]. Additionally, in recent years, some Acinetobacter strains have demonstrated a propensity to acquire resistance to multiple classes of antibiotics, rendering treatment of such hospitalized patients extremely difficult [4]. Furthermore, in pediatric patients, A. baumannii is thought to be the most prevalent organism, which is associated with 
bacteremia, ventilator-associated pneumonia, bronchopulmonary dysplasia, meningitis, and neutropenia $[3,5,6]$.

Currently, our knowledge of the clinical impact of Acinetobacter infections in the pediatric patient population in developing countries and countries with suboptimal infection control resources have been well documented [2, 3, 7-11]. However, invasive Acinetobacter infections associated with large pediatric, academic institutes in developed countries are still poorly defined due to unclear risk factors. Recent studies documented risk factors for invasive infections as catheter insertions, prolonged use of antibiotics, as well as underlying chronic diseases $[5,12]$. It is not clear if these risk factors are universal in all hospital settings, including locations outside of intensive care units. Additionally, improvements in bacterial sequencing, in particular the Acinetobacter rpoB gene, have allowed us to more accurately identify clinically important Acinetobacter species [13-15].

Therefore, the purpose of this study was to retrospectively review cases of invasive Acinetobacter infections occurring within an academic, pediatric setting in a developed country from a clinical perspective to define additional risk factors. Furthermore Acinetobacter strains were sequenced typed and preliminary characterized for motility and biofilm formation/maintenance. We hypothesized that Acinetobacter infections would be restricted to ICU settings and predominantly comprised of strains of $A$. baumannii.

\section{Methods}

\section{Study design}

The study was an observational review of patients with invasive Acinetobacter infections correlated with comparative sequencing of hyper-variable regions of the $r p o B$ gene of Acinetobacter isolates. It was reviewed and approved by the Institutional Review Board of Nationwide Children's Hospital (IRB14-00145). As aggregate patient data was used, individual consent was not obtained. The review population consisted of pediatric patients at a single, large, academic, pediatric institution identified during the years of 2009-2013. Inclusion criteria included pediatric patients less than 21 years old with at least one positive blood, bone, endotracheal, peritoneal, or cerebrospinal fluid culture result positive for Acinetobacter species [all identified as A. baumannii or $A$. baumannii complex by the clinical laboratory using Vitek 2 (bioMerieux, Durham NC) and other phenotypic methods as needed]. Antimicrobial susceptibility testing was performed on all isolates via the Vitek 2 using established breakpoints [16]. Multi-drug resistant isolates (MDR) was defined as isolates non-susceptible to $\geq 1$ antimicrobial agent in $\geq 3$ antimicrobial categories as defined by the joint initiative of the European Centre for Disease Prevention and Control (ECDC) and the Centers for Disease Control and Prevention (CDC) [17]. Study samples represented a convenience sample of existing isolates available in the clinical laboratory. Patients with cystic fibrosis respiratory isolates of Acinetobacter were excluded from the study. Isolates were frozen and stored at $-80{ }^{\circ} \mathrm{C}$.

A comprehensive review of clinical and demographic patient information was performed surrounding positive culture identification. Patient demographics included gender, age, race, county of residence, and insurance type. Information related to determining the source of the infection including the patient's location within the hospital, source of the culture, and susceptibilities were recorded. A $48 \mathrm{~h}$ time period after cultures were obtained was chosen as an initial analysis point based on standard hospital time-based culture practices needed for determining culture results. At this time point antibiotic selection by the practitioner might change based on available antimicrobial susceptibilities. Underlying diagnoses, co-morbidities, secondhand smoke exposure, and vital signs were recorded. Laboratory data included complete blood count with differential, creatinine, liver function tests, as well as the next 5 culture results for each patient. C-reactive protein and erythrocyte sedimentation rates were available on less than $20 \%$ of the cohort and therefore not included. Information regarding the patient's hospital stay included length of stay, febrile status, oxygen use, ventilator use, imaging and invasive procedures, and mortality. Antibiotic treatment including antibiotics given and length taken by the patient along with antibiotic usage in the past 30 days were noted. Home medication including the use of chronic immunosuppressants, antibiotics, and gastric acid suppressants were all recorded.

\section{Comparative sequence analysis of the rpoB gene from clinical Acinetobacter isolates}

Acinetobacter rpoB hyper-variable region sequencing was performed on Acinetobacter isolates according to a previously established protocol [18] with the following modifications. Genomic DNA (gDNA) was prepared from Acinetobacter isolates utilizing the Gentra Puregene Yeast/ Bact. Kit B (Qiagen) according to the manufacturer's protocol. One hundred nanograms of gDNA from each isolate were used in a PCR with one of two sets of primers. The first set of primers, spanning zone 1, were Ac696F (5'TAYCGYAAAGAYTTGAAAGAAG-3') and Ac1093R (5'CMACACCYTTGTTMCCRTGA-3') and the second set of primers, spanning zone 2, were Ac1055F (5'-GTGATAA RATGGCBGGTCGT-3') and Ac1598R (5'-CGBGCRTGC ATYTTGTCRT-3').

DreamTaq DNA polymerase (Thermo Scientific) was used according to manufacturer's protocol with the following thermocycling conditions: 1 cycle at $95{ }^{\circ} \mathrm{C}$ for 
2 min; 30 cycles at $95{ }^{\circ} \mathrm{C}$ for $30 \mathrm{~s}, 55^{\circ} \mathrm{C}$ for $30 \mathrm{~s}$, and $72{ }^{\circ} \mathrm{C}$ for $1 \mathrm{~min} ; 1$ cycle at $72{ }^{\circ} \mathrm{C}$ for $10 \mathrm{~min}$. PCR products were purified using the QiaQuick PCR purification kit (Qiagen) according to the manufacturer's protocol. Clean PCR products were verified by electrophoresis and sent off for sequencing by Eurofins MWG Operon. Raw sequence files were trimmed and edited using MegAlign and EditSeq software applications (DNASTAR). Trimmed sequence files for the two hypervariable regions of a single isolate were combined into a single FASTA file for phylogenetic analysis. The combined FASTA files for each isolate, as well the corresponding sequences from the reference Acinetobacter strains used for phylogenetic analysis, were aligned initially by the Clustal W method utilizing UPGMB for the cluster method and the Kmer4-6 method for the distance measure. Later parameters utilized UPGMB for the cluster method and the Kimora \% identity method for distance measure. Sequence distances were computed with the metric uncorrected pairwise distance and assembled into a phylogenetic tree using the MegAlign Pro software (DNASTAR).

\section{Motility assays}

Twitching motility was investigated per previously described protocols with the following modifications [19]. Twitching plates contained $10 \mathrm{~g}$ tryptone/L and $10 \mathrm{~g}$ agarose/L. Briefly, bacterial strains were grown overnight and a single colony was used to inoculate each twitching plate to the bottom of the petri dish with a sterile wooden applicator stick. Twitching plates were incubated at $37{ }^{\circ} \mathrm{C}$ in a humidified incubator for $16 \mathrm{~h}$. To visualize zones of twitching motility, the agarose was removed, the adherent bacterial population was washed with phosphate buffered saline, and stained with $0.1 \%$ crystal in water. Bacteria positively exhibiting twitching motility were defined by demonstrating a zone of motility of $>10 \mathrm{~mm}$ around the site of inoculation. Surfaceassociated motility was simultaneously assessed per our previously published methodologies [19]. Bacteria positively exhibiting surface-associated motility were defined as strains with a halo growth zone of $>20 \mathrm{~mm}$. Assays were performed in triplicate.

\section{Crystal violet retention assay}

In order to assess the ability of each isolate to form biofilms, the crystal violet retention assay was performed as described previously [20] with minor modifications. Mueller Hinton $(\mathrm{MH})$ broth was inoculated with one bacterial colony and incubated overnight at $37{ }^{\circ} \mathrm{C}$ for approximately $16-18 \mathrm{~h}$. Cultures were subsequently diluted 1:100 in fresh $\mathrm{MH}$ broth and $100 \mu \mathrm{l}$ of diluted culture was added to each well in 96 well microtiter plate and incubated overnight at $37{ }^{\circ} \mathrm{C}$. Adherent cells were washed once with deionized water and stained with $125 \mu \mathrm{l}$ of $0.1 \%$ crystal violet solution for $15 \mathrm{~min}$ at room temperature and washed 5 times with deionized water. Subsequently, dye was released from the cells using $200 \mu \mathrm{l}$ of $95 \%$ ethanol. Absorbance was measured at 595 nm on a Synergy H1 Hybrid Reader spectrophotometer (Biotech, Biotech Instruments, Vermont, USA). The biofilm data represent the average of three independent experiments of triplicate wells.

\section{Statistical analysis}

All analyses were performed using Stata/MP, version 13.1 or GraphPad Prism version 6.03. For all analyses, a $P$ value $<0.05$ was considered statistically significant. Descriptive statistics for continuous variables were presented as medians with $25-75^{\text {th }}$ percentile ranges; and descriptive statistics for categorical variables were presented as proportions. Mann-whitney tests were performed for sum comparisons.

\section{Results \\ Bacterial isolates}

Twenty-four isolates reported as A. baumannii from the clinical microbiology laboratory were recovered during the study period from 22 separate patients. A phylogenetic tree was derived after comparative sequence analysis of hyper-variable regions of the rpoB gene from Acinetobacter isolates using the BioNJ algorithm [21] (Fig. 1). The isolates were predominately composed of $A$. pittii (45.8\%) and A. baumannii (33.3\%). Other identified strains included one strain of $A$. calcoaceticus and two strains of $A$. nosocomialis. Two isolates, 26702 and 33291 were non-identified species. Both cases of Acinetobacter-associated death were caused by $A$. pittii. Notably, $70.0 \%(n=7)$ of the isolates in the last 2 years of the study were also $A$. pittii, representing a predominant shift towards $A$. pittii for the most recent institutional isolates $(30.8 \% A$. pittii the previous three years combined, $n=4)$. There were no reported hospital or unit specific outbreaks of Acinetobacter infection during the study period.

\section{Subject characteristics}

Patient demographics and characteristics are listed in Table 1 . The population was predominantly Caucasian and had a median age of 3.5 years. Identified risk factors for systemic infection are listed in Table 1. There was no consistent underlying primary diagnosis for the patients, as a total of 17 unique primary conditions were found for the 22 patients. Nearly $71 \%$ of subjects had an indwelling central venous catheter source of the first positive culture with a non-significant increase in subjects with $A$. pittii $(72.7 \%)$ compared to A. baumannii (62.5\%). Over $40 \%$ of subjects were on chronic 


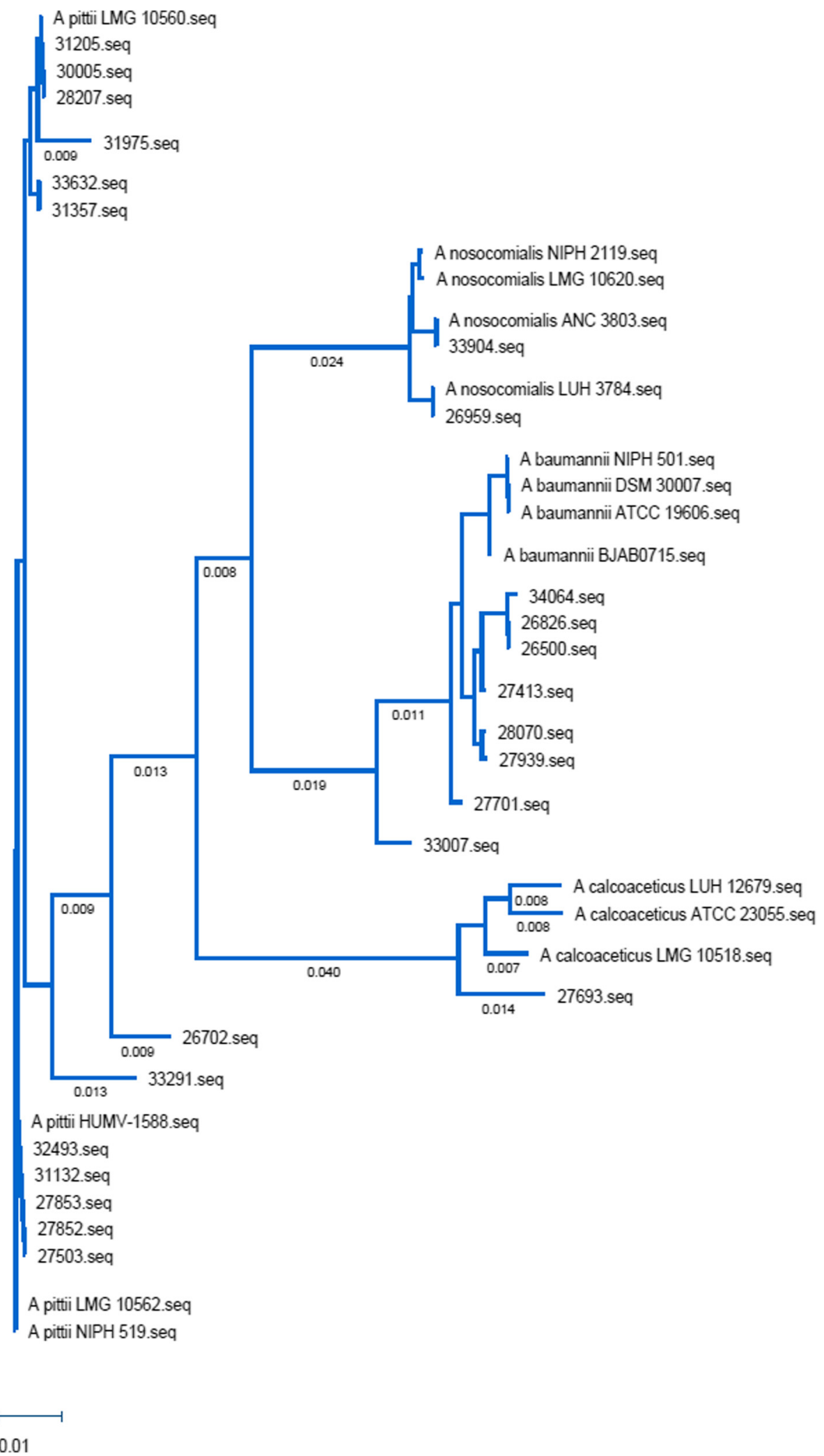

Fig. 1 Phylogenetic Tree of Acinetobacter Clinical Isolates. Acinetobacter rpoB sequences from our collection as well as reference sequences were aligned using Clustal Omega based on the BioNJ algorithm. The tree was built using MegAlign Pro from the DNASTAR suite of programs. Horizontal branch length is a measure of genetic distance 
Table 1 Patient demographics and identified risk factors for Acinetobacter infection

\begin{tabular}{|c|c|c|}
\hline & Percentage / Median & $\begin{array}{l}\text { Range (if median } \\
\text { reported) }\end{array}$ \\
\hline \multicolumn{3}{|l|}{ Patient Demographics } \\
\hline Male & $62.5 \%$ & \\
\hline Caucasian & $79.2 \%$ & \\
\hline Medicaid & $70.8 \%$ & \\
\hline Age (years) & 3.5 & $1.0-13.8$ \\
\hline Body Mass Index (BMI) & 18.0 & $15.8-21.2$ \\
\hline \multicolumn{3}{|l|}{ Source } \\
\hline Blood & $79.2 \%$ & \\
\hline Peritoneal Fluid & $4.2 \%$ & \\
\hline Bone/soft tissue & $8.3 \%$ & \\
\hline Respiratory tract & $8.3 \%$ & \\
\hline \multicolumn{3}{|l|}{ Risk Factors } \\
\hline Previously healthy & $0 \%$ & \\
\hline Central venous catheter & $70.8 \%$ & \\
\hline $\begin{array}{l}\text { Past bone } \\
\text { marrow transplant }\end{array}$ & $20.8 \%$ & \\
\hline $\begin{array}{l}\text { Secondhand } \\
\text { smoke exposure }\end{array}$ & $29.2 \%$ & \\
\hline $\begin{array}{l}\text { Taken antibiotics } \\
\text { in past } 30 \text { days }\end{array}$ & $41.7 \%$ & \\
\hline Chronic antibiotics $>30$ days & $41.7 \%$ & \\
\hline $\begin{array}{l}\text { Chronic } \\
\text { immunosuppressants }\end{array}$ & $37.5 \%$ & \\
\hline Gastroesophageal reflux & $41.7 \%$ & \\
\hline
\end{tabular}

antibiotics due to an immunosuppressive state at the time of Acinetobacter culture positivity, with nearly equal use between $A$. baumannii (50 \%) and A. pittii (45.8 \%) species. Complete data was available for all patients.

\section{Hospital characteristics}

Laboratory data and other characteristics relevant to the patients' stay in the hospital are listed in Table 2. Markers of systemic kidney (creatinine) or liver (ALT, AST) dysfunction were not present for the majority of patients. Median length of stay for subjects was 10.5 days, with a non-significant increase in length of stay for $A$. pittii compared to $A$. baumannii (13.5 vs 3.5 days, $P$ value $=0.13$ ). Only two patients died during hospitalization, both with $A$. pittii infection. There was a significant increase in oxygen use for patients with $A$. pittii isolates compared to $A$. baumannii (54.5\% vs $0 \%$, $P$ value $=0.018)$. Two-thirds of positive cultures were obtained in settings outside of the ICU, however $45.5 \%$ of $A$. pittii isolates were from an ICU setting compared to $25.0 \%$ A. baumannii. Median duration of antibiotic use was 14.0 days, including outpatient completion if subject discharged from hospital. Almost $80 \%$ of repeat cultures were negative for Acinetobacter species within $48 \mathrm{~h}$ of initiating antibiotics. Antibiotic susceptibility patterns for the recovered isolates are shown in Table 3 as the percent of susceptible isolates to a given antibiotic. $72.7 \%$ of A. pittii isolates were pan-susceptible, compared to $62.5 \%$ of $A$. baumannii isolates $(P$ value $=$ $0.99)$. Additionally, the clinical laboratory designated $17 \%(n=4)$ of the isolates as MDR at the time of initial isolation, with three of these four strains from the $A$. baumannii group. Tobramycin demonstrated the least overall resistance (91.7\% strains susceptible, Table 3). Antibiotic selection for the first $48 \mathrm{~h}$ after clinical onset of infection and the final treatment course are shown in Table 4. Gentamicin was the most commonly used antibiotic for the full treatment course, although a wide variety of antibiotics were used during the first $48 \mathrm{~h}$ after onset of positive cultures (Additional file 1: Table S1).

\section{Acinetobacter isolate motility}

Twitching and surface-associated motility (swarming) have been shown to be distinct phenotypes for medically relevant Acinetobacter [19, 22], but have not been described for $A$. pittii. All isolated strains were assessed for motility characteristics (Fig. 2a). Surface-associated motility was observed in $63.6 \%$ of the $A$. pittii strains, and $62.5 \%$ of the $A$. baumannii strains ( $P$ value $=0.99$ ). Twitching motility was observed in $72.7 \%$ of the $A$. pittii strains, and $50 \%$ of the $A$. baumannii strains ( $P$ value $=0.37)$. Both phenotypes were present in $45.5 \%$ of the $A$. pittii strains, and only $25 \%$ of the $A$. baumannii, with the four most recently isolated $A$. pittii strains harboring both phenotypes. The $A$. calcoaceticus strain demonstrated twitching, but not swarming motility.

\section{Biofilm formation}

The production of biofilms allow for bacterial persistence and adherence to medical devices and vascular lines [23]. Because over $70 \%$ of our isolates were associated with central line infections, we wanted to determine if there were any differences in the capability of the isolates to form biofilms, which may predispose to local strain emergence on medical devices. To quantitate Acinetobacter strain biofilm capabilities, individual strains were tested for their ability to adhere to microtiter plates as determined by crystal violet stain retention. A. pittii isolates had significantly higher median biofilm production compared to $A$. baumannii isolates (Fig. $2 \mathrm{~b}, P$ value $=$ 0.0071. Furthermore, both $A$. nosocomialis isolates (\#26959, \# 33904) demonstrated an intermediate ability to form biofilms as determined by crystal violet retention, however, the A. calcoaceticus isolate (\#27693) demonstrated a minimal ability to form a biofilm (Fig. 2a). Overall, $75 \%$ of all isolates were able to form biofilms. 
Table 2 Patient laboratory parameters and hospital characteristics

\begin{tabular}{|c|c|c|c|}
\hline & All isolates Percent/Median (Range) & A. baumannii & A. pittii \\
\hline \multicolumn{4}{|l|}{ Laboratory Data } \\
\hline White blood cell $\left(\mathrm{k} / \mathrm{mm}^{3}\right)$ & $7.7(1.6-14.3)$ & $7.7(1.2-11.0)$ & $6.8(1.5-20.9)$ \\
\hline Hemoglobin (g/dL) & $9.2(7.8-9.9)$ & $9.0(7.6-9.3)$ & $8.8(7.8-11.1)$ \\
\hline Neutrophils & $60.0 \%(44.0-76.0)$ & $62.0 \%(26.8-76.3)$ & $57.0 \%(49.5-69.5)$ \\
\hline Creatinine (mg/dL) & $0.45(0.32-0.65)$ & $0.44(0.37-0.65)$ & $0.35(0.24-0.65)$ \\
\hline ALT (IU/L) & $37.5(18.0-143.3)$ & $13.0(8.0-18.0)$ & $135.0(37.5-157.5)$ \\
\hline AST (IU/L) & $45.5(38.5-93.5)$ & $39.0(37.0-41.0)$ & $71.0(45.0-177.0)$ \\
\hline \multicolumn{4}{|l|}{ Hospital Visit } \\
\hline $\begin{array}{l}\text { Length of stay (days) } \\
\text { Location of positive culture }\end{array}$ & $10.5(3.3-67.5)$ & $3.5(1.5-16.3)$ & $13.5(5.0-82.5)$ \\
\hline Intensive care unit (ICU) & $33.3 \%$ & $25.0 \%$ & $45.5 \%$ \\
\hline Hematology / Oncology unit & $33.3 \%$ & $37.5 \%$ & $36.4 \%$ \\
\hline Emergency / Outpatient & $33.3 \%$ & $37.5 \%$ & $18.1 \%$ \\
\hline Oxygen use & $33.3 \%$ & $0 \%$ & $54.5 \%$ \\
\hline Ventilator use & $37.5 \%$ & $18.2 \%$ & $45.5 \%$ \\
\hline Mortality & $8.3 \%$ & $0 \%$ & $18.2 \%$ \\
\hline Antibiotic duration (days) & $14.0(9.3-14.0)$ & $14.0(10.0-19.0)$ & $14.0(6.0-14.0)$ \\
\hline Follow up culture negative ${ }^{a}$ & $79.9 \%$ & $87.5 \%$ & $81.8 \%$ \\
\hline
\end{tabular}

${ }^{\mathrm{a}}$ Results of the next bacterial culture within $48 \mathrm{~h}$ of antibiotic initiation

\section{Clinical characteristics based on phenotypic expression}

After Acinetobacter isolate motility and biofilm formation studies were performed, isolates were than characterized for relevant clinical outcomes based on the percentage of isolates expressing each phenotype (Table 4). There was no difference in biofilm production between central lineassociated and non-central line-associated isolates (median absorbance 0.35 vs $0.42, P$ value $=0.62$ ), although there was a non-statistically significant increase in nonbiofilm producing isolates associated with central line infections $(85.7$ vs $64.7 \%, P$ value $=0.38)$. However, all isolates that demonstrated persistent bacteremia at $48 \mathrm{~h}$ were biofilm-producers. Multi-drug resistance was present in both biofilm and non-biofilm producing isolates, but MDR isolates were predominantly non-biofilm producers ( 42.9 vs $5.9 \%, P$ value $=0.027$ ). Chronic immunosuppressant use was greater in non-biofilm producing isolates, but this did not reach statistical significance (71.4 vs $29.4 \%, P$ value $=0.06$ ). There was no difference in the requirement for ICU care, secondhand smoke exposure, or use of proton pump inhibitors for gastroesophageal reflux based on biofilm formation. There were no discernible differences between twitching and swarming isolates in any of the clinical characteristics.

\section{Discussion}

Members of the Acinetobacter calcoaceticus-baumannii complex are regarded as opportunistic human pathogens of increasing relevance worldwide due in part to the emergence of multiply-drug resistant strains; however, the description of pathogenic $A c b$ members other than A. baumannii remains limited due in part technological limitations with current clinical laboratory identification methods [24]. Although emerging methods such as

Table 3 Antibiotic susceptibility patterns as percentage of susceptible Acinetobacter isolates

\begin{tabular}{llll}
\hline & All isolates $(\%, \mathrm{n})$ & A. pittii $(\%, \mathrm{n})$ & A. baumannii $(\%, \mathrm{n})$ \\
\hline Ceftazidime & $79.2,19$ & $90.1,10$ & $62.5,5$ \\
Ciprofloxacin & $87.5,21$ & 100,11 & $62.5,5$ \\
Gentamicin & $83.3,20$ & $90.1,10$ & $62.5,5$ \\
Meropenem & $87.5,21$ & 100,11 & $62.5,5$ \\
Piperacillin-Tazobactam & $87.5,21$ & 100,11 & $62.5,5$ \\
Trimethoprim-sulfamethoxazole & $83.3,20$ & $90.1,10$ & $75.0,6$ \\
Tobramycin & $91.7,22$ & 100,11 & $75.0,6$ \\
\hline
\end{tabular}


Table 4 Phenotypic characteristics of Acinetobacter isolates association with clinical factors

\begin{tabular}{|c|c|c|c|c|c|c|c|c|c|}
\hline & $\begin{array}{l}\text { Biofilm-forming } \\
(n=17)\end{array}$ & $\begin{array}{l}\text { Non-biofilm- } \\
\text { forming }(n=7)\end{array}$ & $P$ value & $\begin{array}{l}\text { Swarming } \\
\text { motility }(n=16)\end{array}$ & $\begin{array}{l}\text { Non-swarming } \\
\text { motility }(n=8)\end{array}$ & $P$ value & $\begin{array}{l}\text { Twitching } \\
\text { motility }(n=14)\end{array}$ & $\begin{array}{l}\text { Non-twitching } \\
\text { motility }(n=10)\end{array}$ & $P$ value \\
\hline Age (years) & $4.0(0.4-11.5)$ & $3.0(2.0-19.0)$ & 0.84 & $4.5(1.0-16.8)$ & $3.5(0.9-4.0)$ & 0.37 & $3.5(0.6-17.5)$ & $3.5(1.5-6.3)$ & 0.57 \\
\hline $\begin{array}{l}\text { Central line } \\
\text { infection }\end{array}$ & $64.7 \%$ & $85.7 \%$ & 0.38 & $68.8 \%$ & $66.7 \%$ & 0.99 & $71.4 \%$ & $70.0 \%$ & 0.99 \\
\hline Bacteremia at $48 \mathrm{~h}$ & $17.6 \%$ & $0.0 \%$ & 0.53 & $12.5 \%$ & $12.5 \%$ & 0.99 & $14.3 \%$ & $10.0 \%$ & 0.99 \\
\hline MDR & $5.9 \%$ & $42.9 \%$ & 0.027 & $18.8 \%$ & $12.5 \%$ & 0.80 & $21.4 \%$ & $10.0 \%$ & 0.48 \\
\hline ICU & $35.3 \%$ & $28.6 \%$ & 0.76 & $37.5 \%$ & $25.0 \%$ & 0.56 & $42.9 \%$ & $20.0 \%$ & 0.26 \\
\hline Abx in past 30 days & $47.1 \%$ & $28.6 \%$ & 0.43 & $37.5 \%$ & $50.0 \%$ & 0.67 & $42.9 \%$ & $40.0 \%$ & 0.99 \\
\hline Immuno-suppressant & $29.4 \%$ & $71.4 \%$ & 0.06 & $43.8 \%$ & $37.5 \%$ & 0.78 & $42.9 \%$ & $40.0 \%$ & 0.99 \\
\hline Length of stay (days) & $17.0(4.5-80.0)$ & $5.0(3.0-20.0)$ & 0.210 .99 & $18.5(4.3-63.3)$ & $5.0(3.3-35)$ & 0.32 & $11.0(3.0-97.5)$ & $10.5(4.5-27.3)$ & 0.92 \\
\hline SHS exposure & $23.5 \%$ & $28.6 \%$ & 0.99 & $34.1 \%$ & $53.5 \%$ & 0.13 & $28.6 \%$ & $20.0 \%$ & 0.99 \\
\hline GER & $35.3 \%$ & $28.6 \%$ & & $43.8 \%$ & $12.5 \%$ & 0.14 & $35.7 \%$ & $30.0 \%$ & 0.99 \\
\hline
\end{tabular}

Data are expressed as a percentage of cases with medians and ranges

$A b x$ antibiotic use in 30 days prior to infection; GER gastroesophageal reflux; ICU Intensive care unit care required; MDR multi-drug resistance with isolates non-susceptible to $\geq 1$ antimicrobial agent in $\geq 3$ antimicrobial categories; SHS secondhand smoke

MALDI-ToF analysis may aid in clinical laboratorybased identification $A c b$ members for the future, these methods have not become the gold-standard even in developed countries. In this study we report the emergence of $A$. pittii strains from pediatric patients with invasive infections, along with a relatively low mortality rate.

Outbreaks of $A$. pittii have only been previously reported to a low extent and were thought to be rare in pediatric settings in the United States $[5,8,9,25]$. This may be attributed to the fact that most of the common clinical laboratory identification methods do not reliably differentiate between members of the $A c b$; hence, many publications may in fact be referring to all members of the $A c b$ when specifically referencing $A$. baumannii $[5,24]$.
Importantly, in the documented cases where $A$. pittii has been reported, it can be more commonly recovered from samples than A. baumannii [8], but this is not the case in all settings. In our isolate collection, there was an emergence of $A$. pittii over the last two years of the study period, indicating a shift towards $A$. pittii over $A$. baumannii. This finding will require further follow-up due to the short time-frame of study follow-up. The significant rise in the number of isolates of $A$. pittii in a pediatric population also signifies a potential new trend to be cognizant of for clinicians and researchers, due largely to differences in antibiotic resistance profiles between species. Notably, we did not see a difference in basic microbiologic characteristics such as surface motility between the $A$. pittii and $A$. baumannii strains. However, the majority of the most
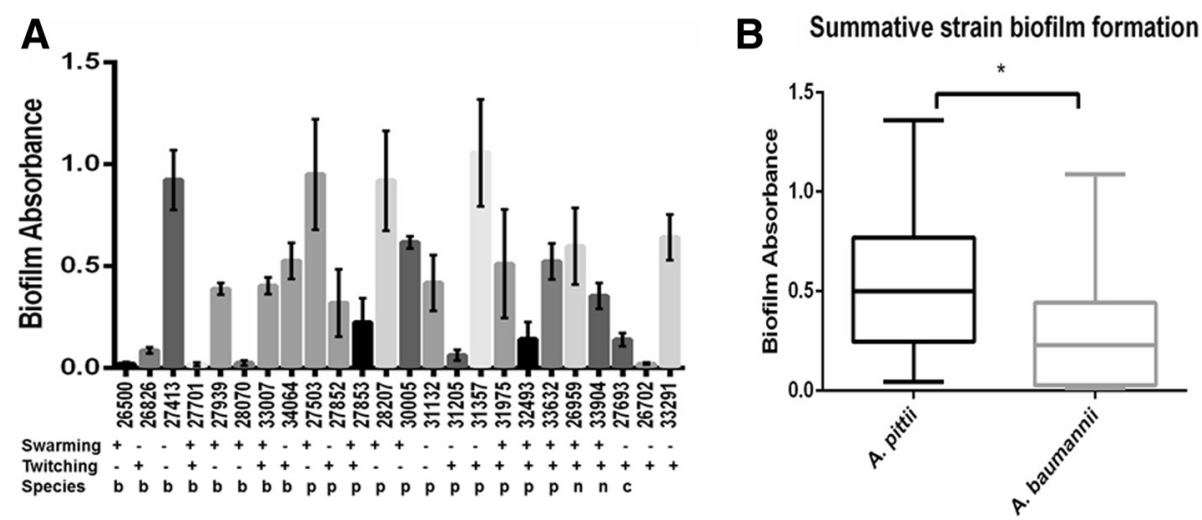

Fig. 2 Bacterial isolate characteristics. a Individual isolate results of biofilm and twitching and surface-associated motility assays with grouping by species. The level of biofilm formation was determined by measuring the absorbance at $595 \mathrm{~nm}$ post crystal violet staining. Error bars represent the standard deviation of the mean of three independent experiments. Twitching and surface-associated motility are characterized as positive or negative based on experimental methods. All experiments performed in triplicate. Strains are designated as: "b" = baumannii, " $p$ " = pittii, " $n$ " = nosocomialis, " $\mathrm{C}$ " = calcoaceticus, two strains are undesignated. $\mathbf{b}$ Box and whiskers plot demonstrating sum of median biofilm production with minimum and maximum error bars for $A$. pittii and $A$. baumannii strains from Fig. 2 a, $P$ value $=0.018$ 
recent $A$. pittii strains did display both twitching and surface-associated motility phenotypes, indicating the likely expression of a functional type IV pilus as well as changing local population dynamics. Additionally, A pittii isolates averaged more robust biofilm production when compared to $A$. baumannii isolates, indicating another potential pathogenic mechanism for the increasing $A$ pittii prevalence. However, laboratory biofilm studies may not reflect human in vivo biofilm formation, therefore further studies are warranted in this area for the future.

Despite previous reports of high mortality associated with antibiotic resistant Acinetobacter strains [5, 7], in our sampling only 2 out of 24 cases were associated with patient deaths. Notably, both deaths were from A. pittii, again in contrast with the existing pediatric literature on A. baumannii-associated mortality, and the antibiotic resistance patterns observed. We postulate that one difference in the overall low mortality rates could be the relative sensitivity to the aminoglycoside antibiotics coupled with prompt administration, as this class of antibiotic was utilized in the first $48 \mathrm{~h}$ of a suspected infection in half of the reported cases. There was also a low prevalence of MDR strains. Both of these factors may have contributed to the fact that nearly $80 \%$ of the strains were cleared from the systemic circulation within $48 \mathrm{~h}$ of initiating antibiotics. Persistent bacteremia was noted to be associated with biofilm-producing isolates, which is of importance when assessing therapeutic options for persistent Acinetobacter infections. Additionally, compared to other reported pediatric Acinetobacter infections, the isolated strains demonstrated lower overall morbidity as reflected by fewer intensive care requirements and less multiple organ involvement. Much of the published pediatric literature focuses on neonatal intensive care units with multiple Acinetobacter infections [3, 8-12]. However, in our sample, the median age was 3.5 years with no reported neonatal infections. We were unable to assess for the role of existing institutional infection control bundles in preventing ICU-associated infections during this retrospective study. Therefore, continued work will be needed to address potential epidemiologic factors associated with pediatric Acinetobacter infections in the United States.

Other species of Acinetobacter are increasingly recognized to cause disease in humans such as $A$. bereziniae [26], further supporting the assumption that improved species identification of Acinetobacter infections may be needed to accurately follow emerging infectious trends from an epidemiologic level. These results further highlight the medical relevance of the Acinetobacter genus as whole, where an encounter between a patient with a compromised immune system and a species of Acinetobacter, regardless of its characterized pathogenicity can result in infection.
We acknowledge that this study has several limitations. First, the study was retrospective in nature, therefore limiting the availability of some clinical information. Second, the small sample size, 5-year time frame, and single-center setting limits the generalizability of the findings and analysis of isolate phenotypic characteristics in relation to clinical outcomes. However, the samples may be more representative of U.S. pediatric academic centers than previous reports from developing countries. Finally, the clinical laboratory did not report antibiotic susceptibilities for all classes of antibiotics for the four isolates obtained in the first year of the study.

\section{Conclusions}

In summary, knowledge of pediatric Acinetobacter infections has been limited to date by clinical laboratory identification methods, which may presume Acinetobacter induced infections to be caused by A. baumannii. A. pittii was discovered to be the dominant local clinical strain expressing a variety of phenotypic characteristics including 2 fatalities. The isolated strains in this study had limited overall long-term morbidity or mortality in a pediatric population, but the occurrence of more MDR strains in the future is a distinct possibility, leading to the need for continued research and accurate species identification.

\section{Additional files}

Additional file 1: Table S1. Frequency of antibiotic regimens utilized during treatment of infected patients. Description: Breakdown of antibiotic choices utilized clinically during the first $48 \mathrm{~h}$ of suspected Acinetobacter infection as well as the final treatment choices. (DOC $33 \mathrm{~kb}$ )

Additional file 2: $r p \circ B$ sequences from Acinetobacter isolates. Description: Full rpoB sequences from each Acinetobacter isolate in the study. (DOC $41 \mathrm{~kb}$ )

\section{Abbreviations}

$A c b$, Acinetobacter calcoaceticus-baumannii; gDNA, genomic DNA; ICU, intensive care unit; $\mathrm{MH}$, Mueller Hinton

\section{Acknowledgements}

The authors thank the NCH clinical microbiology laboratory staff for assistance with isolates.

\section{Funding}

Funding was provided through $\mathrm{NCH}$ internal funding to BK.

Availability of data and materials

The Acinetobacter isolate sequence files have been included as Additional file 2.

Authors' contributions

AJ and $\mathrm{CMH}$ equally participated in study design, data acquisition, experimental completion, data analysis, and drafting and editing the manuscript. KA and CS participated in biofilm and twitching experimental completion and $\mathrm{MH}$ participated in isolate identification. AL participated in study design, isolate acquisition, analysis, and manuscript editing. RM participated in study design, analysis, and manuscript editing. BK designed the study, had oversight of data acquisition and analysis, experimental performance, and manuscript writing and editing. All authors have read and approve the final version of the manuscript.

Competing interests

The authors declare that they have no competing interests. 


\section{Consent for publication}

Not applicable.

\section{Ethics approval and consent to participate}

This study was reviewed and approved by the Institutional Review Board of Nationwide Children's Hospital (IRB14-00145). As aggregate patient data was used, individual consent was not obtained per this approval.

\section{Author details}

${ }^{1}$ Center for Microbial Pathogenesis, The Research Institute at Nationwide Children's Hospital, Columbus, OH, USA. ${ }^{2}$ Biomedical Sciences Graduate Program, College of Medicine, The Ohio State University, Columbus, OH, USA. ${ }^{3}$ Department of Laboratory Medicine, Nationwide Children's Hospital, Columbus, OH, USA. ${ }^{4}$ Nationwide Children's Hospital, Section of Pulmonary Medicine, 700 Children's Drive, Columbus, OH 43205, USA.

Received: 21 October 2015 Accepted: 14 June 2016

\section{Published online: 22 July 2016}

\section{References}

1. Bergogne-Berezin E, Towner KJ. Acinetobacter spp. as nosocomial pathogens: microbiological, clinical, and epidemiological features. Clin Microbiol Rev. 1996;9(2):148-65.

2. Peleg AY, Seifert $H$, Paterson DL. Acinetobacter baumannii: emergence of a successful pathogen. Clin Microbiol Rev. 2008:21(3):538-82.

3. Hu J, Robinson JL. Systematic review of invasive Acinetobacter infections in children. Can J Infect Dis Med Microbiol. 2010;21(2):83-8.

4. Afzal-Shah M, Livermore DM. Worldwide emergence of carbapenem-resistant Acinetobacter spp. J Antimicrob Chemother. 1998:41(5):576-7.

5. Wisplinghoff $H$, Paulus $T$, Lugenheim M, Stefanik D, Higgins PG, Edmond $M B$, Wenzel RP, Seifert H. Nosocomial bloodstream infections due to Acinetobacter baumannii, Acinetobacter pittii and Acinetobacter nosocomialis in the United States. J Infect. 2012;64(3):282-90.

6. Lohmann P, Luna RA, Hollister EB, Devaraj S, Mistretta TA, Welty SE, Versalovic J. The airway microbiome of intubated premature infants: characteristics and changes that predict the development of bronchopulmonary dysplasia. Pediatr Res. 2014;76(3):294-301.

7. Macao P, Lopes JC, Oliveira H, Oliveira G, Rodrigues F. [Health care associated multidrug-resistant bacteria in a pediatric hospital: five year experience]. Acta Medica Port. 2013;26(4):385-91.

8. Wang X, Chen T, Yu R, Lu X, Zong Z. Acinetobacter pittii and Acinetobacter nosocomialis among clinical isolates of the Acinetobacter calcoaceticusbaumannii complex in Sichuan, China. Diagn Microbiol Infect Dis. 2013;76(3):392-5.

9. Saleem AF, Ahmed I, Mir F, Ali SR, Zaidi AK. Pan-resistant Acinetobacter infection in neonates in Karachi, Pakistan. J Infect Dev Ctries. 2010;4(1):30-7.

10. Balkhy HH, Bawazeer MS, Kattan RF, Tamim HM, Al Johani SM, Aldughashem FA, Al Alem HA, Adlan A, Herwaldt LA. Epidemiology of Acinetobacter spp.associated healthcare infections and colonization among children at a tertiary-care hospital in Saudi Arabia: a 6-year retrospective cohort study. Eur J Clin Microbiol Infect Dis. 2012;31(10):2645-51.

11. Litzow JM, Gill CJ, Mantaring JB, Fox MP, MacLeod WB, Mendoza M, Mendoza S, Scobie R, Huskins CW, Goldman DA et al. High frequency of multidrug-resistant gram-negative rods in 2 neonatal intensive care units in the Philippines. Infect Control Hosp Epidemiol. 2009;30(6):543-9.

12. Ekinci F, Bayram N, Devrim I, Apa H, Gulfidan G, Gunay I. Estimating risk factors for acinetobacter bacteremia in pediatric settings. Braz J Infect Dis. 2013;17(4):505-6.

13. Gundi VA, Dijkshoorn L, Burignat S, Raoult D, La Scola B. Validation of partial rpoB gene sequence analysis for the identification of clinically important and emerging Acinetobacter species. Microbiology. 2009;155(Pt 7):2333-41.

14. Khosravi AD, Sadeghi P, Shahraki AH, Heidarieh P, Sheikhi N. Molecular methods for identification of acinetobacter species by partial sequencing of the rpoB and 16S rRNA genes. J Clin Diagn Res. 2015;9(7):DC09-13.

15. Fitzpatrick MA, Ozer E, Bolon MK, Hauser AR. Influence of ACB complex genospecies on clinical outcomes in a U.S. hospital with high rates of multidrug resistance. J Infect. 2015;70(2):144-52.

16. Clinical Laboratory Standards Institute. Performance standards for antimicrobial susceptibility testing, M100-S23. Wayne: Clinical and Laboratory Standards Institute; 2013.
17. Magiorakos AP, Srinivasan A, Carey RB, Carmeli Y, Falagas ME, Giske CG, Harbarth S, Hindler JF, Kahlmeter G, Olsson-Liljequist B et al. Multidrug-resistant, extensively drug-resistant and pandrug-resistant bacteria: an international expert proposal for interim standard definitions for acquired resistance. Clin Microbiol Infect. 2012;18(3):268-81.

18. La Scola B, Gundi VA, Khamis A, Raoult D. Sequencing of the rpoB gene and flanking spacers for molecular identification of Acinetobacter species. J Clin Microbiol. 2006;44(3):827-32.

19. Harding CM, Tracy EN, Carruthers MD, Rather PN, Actis LA, Munson RS, Jr. Acinetobacter baumannii strain M2 produces type IV pili which play a role in natural transformation and twitching motility but not surface-associated motility. MBio. 2013;4(4). doi:10.1128/mBio.00360-13.

20. OToole GA, Pratt LA, Watnick PI, Newman DK, Weaver VB, Kolter R. Genetic approaches to study of biofilms. Methods Enzymol. 1999;310:91-109.

21. Gascuel O. BIONJ: an improved version of the NJ algorithm based on a simple model of sequence data. Mol Biol Evol. 1997;14(7):685-95.

22. Eijkelkamp BA, Stroeher UH, Hassan KA, Papadimitrious MS, Paulsen IT, Brown $\mathrm{MH}$. Adherence and motility characteristics of clinical Acinetobacter baumannii isolates. FEMS Microbiol Lett. 2011;323(1):44-51.

23. Percival SL, Suleman L, Vuotto C, Donelli G. Healthcare-associated infections, medical devices and biofilms: risk, tolerance and control. J Med Microbiol. 2015;64(Pt 4):323-34.

24. Viehman JA, Nguyen MH, Doi Y. Treatment options for carbapenemresistant and extensively drug-resistant Acinetobacter baumannii infections. Drugs. 2014;74(12):1315-33.

25. van Dessel H, Kamp-Hopmans TE, Fluit AC, Brisse S, de Smet AM, Dijkshoorn L, Troelstra A, Verhoef J, Mascini EM. Outbreak of a susceptible strain of Acinetobacter species 13 (sensu Tjernberg and Ursing) in an adult neurosurgical intensive care unit. J Hosp Infect. 2002;51(2):89-95.

26. Bonnin RA, Ocampo-Sosa AA, Poirel L, Guet-Revillet H, Nordmann P. Biochemical and genetic characterization of carbapenem-hydrolyzing beta-lactamase OXA-229 from Acinetobacter bereziniae. Antimicrob Agents Chemother. 2012;56(7):3923-7.

\section{Submit your next manuscript to BioMed Central and we will help you at every step:}

- We accept pre-submission inquiries

- Our selector tool helps you to find the most relevant journal

- We provide round the clock customer support

- Convenient online submission

- Thorough peer review

- Inclusion in PubMed and all major indexing services

- Maximum visibility for your research

Submit your manuscript at www.biomedcentral.com/submit
) Biomed Central 